\title{
Multiple House Dust Mite Allergen- Sensitization Profiles in Children with Allergic Asthma
}

\author{
Jennifer Maries G. Yap ${ }^{1}$, Maricar W. Ching ${ }^{2,3^{*}}$, Cristan Q. Cabanilla ${ }^{4}$ and John Donnie A. Ramos ${ }^{1,5}$ \\ ${ }^{1}$ Biological Sciences Department, College of Science, University of Santo Tomas, Philippines \\ ${ }^{2}$ Department of Biological Sciences, College of Science and Technology, Centro Escolar University, Philippines \\ ${ }^{3}$ The Graduate School, Centro Escolar University, Philippines \\ ${ }^{4}$ Philippine Children's Medical Center, Quezon City, Philippines \\ ${ }^{5}$ Research Center for the Natural and Applied Sciences, University of Santo Tomas, Philippines
}

*Corresponding author: Maricar W. Ching, Department of Biological Sciences, College of Science and Technology Centro Escolar University, 1005, Manila, Philippines, Tel: 6327356861; Fax: 6327354451; E-mail: cindywiscoching@gmail.com

Rec date: Apr 25, 2014, Acc date: May 30, 2014, Pub date: June 05, 2014

Copyright: (c) 2014 Yap JMG, et al. This is an open-access article distributed under the terms of the Creative Commons Attribution License, which permits unrestricted use, distribution, and reproduction in any medium, provided the original author and source are credited.

\begin{abstract}
Background: Allergic asthma is a common chronic inflammatory disorder which affects populations, with increasing prevalence among children. The risk of developing asthma is uncertain but depends on the interaction of environmental and genetic factors. The role of house dust mite (HDM) allergen exposure in the development of sensitization and asthma remains unclear.
\end{abstract}

Objective: This study determined the sensitization profiles of common HDM species in a population of asthmatic children, which is essential in the development of highly specific and accurate diagnostic and therapeutic strategies for asthma in children.

Methods: The immunoglobulin E-binding activity of allergens from the HDM species Blomia tropicalis (Bt), Dermatophagoides farina (Df), and Dermatopahagoides pteronyssinus (Dp) were determined in 250 age- and sexmatched paediatric allergic asthma and non-atopic Filipino subjects using Enzyme-linked immunosorbent assay and western blot analysis.

Results: Majority of the allergic asthma patients tested were sensitized with multiple allergens from different HDM species where $33 \%$ exhibited sensitizations to any two HDM species and $26 \%$ were sensitized with allergens from three HDMs. HDM allergens of different molecular weights bind to $\mathrm{IgE}$ in allergic asthma patients tested. In addition a significant correlation was observed between total IgE and HDM-specific IgE level among allergic asthma patients $(B t p$-value $=0.038$; $D f$-value $=0.045$; $D p p$-value $=0.003$ ), suggesting a significant contribution of dust mite allergens in the up-regulation of total serum IgE levels of allergic asthma patients.

Conclusion: The results obtained in this study suggest that the HDM species $B t, D p$, and $D f$ are important sources of allergens that trigger multiple sensitization in children with allergic asthma in the Filipino population. The incorporation of $B t, D p$, and $D f$ allergens in the panel of diagnostic allergens for HDM allergy and allergic asthma is highly recommended.

Keywords: Allergy; Asthma; Total IgE; House dust mite-specific IgE

\section{Introduction}

Allergy is a hypersensitivity reaction initiated by immunological mechanisms characterized by the production of elevated levels of allergen-specific immunoglobulin E (IgE) [1]. Allergic asthma is one of the common chronic respiratory disorders that affect approximately 300 million people of different age worldwide with an increasing prevalence among children [2]. The risks of developing asthma are still uncertain but depend on different complex interaction of hereditary and environmental factors. House dust mite (HDM) allergen exposure is a significant trigger of exacerbations for many asthmatic patients [3]. More than $90 \%$ of children with asthma develop specific IgE sensitization against allergens from HDM, hence, dust mite allergy is strongly associated with asthma [4-6]. The three most common species that is associated with the development of asthma and other allergic diseases are Dermatophagoides pteronyssinus (Dp), Dermatophagoides farinae (Df), and Blomia tropicalis (Bt) [7].

IgE cross reactivity is a common feature of mite allergens, especially in those from taxonomically related species. In vitro cross reactivity studies between Dermatophagoides species and extracts of other mite species showed that mites share common and species-specific allergens [8]. As a result, multiple sensitizations to dust mite species Dermatophagoides sp. and Blomia sp. allergens are often observed among asthmatic children living in tropical and subtropical countries including Philippines [9-11].

Understanding the sensitization profiles of common HDM species in a population of asthmatic children is invaluable in understanding the pathogenesis of HDM allergy and is important in the development of highly specific and accurate diagnostic and therapeutic strategies for 
asthma in children. Sensitization to multiple allergens from different HDM species among asthmatic children is presented in this paper.

\section{Materials and Methods}

\section{Study design and subjects}

The study utilized a case-control method employing 125 age- and sex- match paired allergic asthma patients and non-asthmatic control subjects with no history of allergy. Subjects were recruited from the Philippine Children's Medical Center (PCMC). The PCMC Institutional Review Board approved the study design, conduct of sampling, and experimental protocols. In addition, informed consents and assents were obtained from the patients.

An initial screening was conducted utilizing standardized questionnaires of the International Study of Asthma and Allergy in Childhood (ISAAC) and the International Primary Care Airways Group (IPAG) for recruitment of subjects. Cases were defined in the study as physician-diagnosed asthmatic patient (with asthma alone, concurrent with allergic rhinitis or atopic dermatitis or both) with elevated levels of serum total $\operatorname{IgE}(\geq 100 \mathrm{IU} / \mathrm{mL}$ ) with at least one type of house dust mite (HDM)-specific $\operatorname{IgE}(\geq 50 \mathrm{IU} / \mathrm{mL})$ while non-atopic controls were defined as non-asthmatic subjects without any history of allergy and serum total IgE level of $<100 \mathrm{IU} / \mathrm{mL}$ and HDM-specific IgE level $<50 \mathrm{IU} / \mathrm{mL}$. Furthermore both cases and controls must be (1) naturally born Filipino; (2) unrelated to individual already participated in the study; (3) was born and living in Luzon Island; and (4) aged 6 months to 18 years old at time of study.

\section{Clinical protocol and Phenotyping}

Five milliliters of blood was extracted from the subjects by venipuncture. Blood serum samples were isolated by centrifugation at 10,000 rpm for 10 minutes. Total serum IgE and HDM-specific IgE were quantitated using Enzyme-Linked Immunosorbent Assay (ELISA). IgE concentration was expressed as $\mathrm{IU} / \mathrm{mL}$ and was $\log$ transformed to normalize distribution.

\section{Enzyme-Linked Immunosorbent Assay}

Enzyme Linked Immunosorbent Assay (ELISA) was used to evaluate the profile of sensitization of the allergic asthma patients and non-atopic control sera against allergen extracts from 3 HDM species D. Farinae, D. Pteronyssinus, and B. tropicalis. Briefly, for sandwich ELISA $10 \mu \mathrm{g} / \mathrm{mL}$ of unlabelled anti-human IgE (Pharmingen, CA, USA) and for indirect ELISA $10 \mu \mathrm{g} / \mathrm{mL}$ of the HDM aqueous extracts were coated onto ELISA plates overnight at $4^{\circ} \mathrm{C}$ using $50 \mu \mathrm{L}$ of $0.1 \mathrm{M}$ $\mathrm{NaHCO} 3, \mathrm{pH}$ 8.3. Plates were blocked with $1 \%$ BSA (Sigma) in Phosphate Buffered Saline with $0.05 \%$ Tween 20 (PBS-T) for 1 hour at room temperature. ELISA plates were incubated overnight with $5 \mathrm{x}$ diluted human sera, then for 1 hour at room temperature with biotinylated anti-human IgE (Pharmingen, CA, USA) diluted 1000x in blocking buffer. Plates were incubated with 2000x dilution of ExtrAvidin-Alkaline phosphatase conjugate (Sigma) for 1 hour. Finally, colorimetric reaction was performed using p-nitrophenyl phosphate (Sigma). Absorbance was read at $405 \mathrm{~nm}$ an ELISA reader (BioTek). Human IgE (Pharmingen) was used as a standard per plate in the calculation of IgE concentration.

\section{Sodium Docecyl Sulfate- Polyacrylamide Gel Electrophoresis}

Aqueous HDM extracts were analyzed by Sodium Dodecyl SulfatePolyacrylamide Gel Electrophoresis (SDS-PAGE). Protein samples were mixed with $2 \mathrm{X}$ loading buffer and were boiled for 5 minutes. Samples were separated on a $15 \%$ Tris-Glycine gel using the Mini PROTEAN electrophoresis system (BioRad, Hercules, CA, USA). Gel was run at 110 Volts for 75 minutes. Broad Range Marker (BioRad, Hercules, CA, USA) was used as standard. SDS-PAGE gel was stained with Coomassie brilliant blue R-250 (BDH laboratory Supplies, Poole, England).

\section{Western blot analysis}

The IgE reactivity of selected sera samples to specific allergens found in the three HDM extracts was determined by western blot analysis. In brief, $\mathrm{Bt}, \mathrm{Dp}$, and $\mathrm{Df}$ aqueous extracts were electrophoresed on a 15\% Tris-Glycine gel and electroblotted onto Hybond-C Nitrocellulose membrane (Amersham Life Sciences, Buckinghamshire, England) using the MiniProtean 3 cell (BioRad, Hercules, CA, USA) at $120 \mathrm{~V}$ for 60 minutes. The membrane was blocked with $5 \%$ skimmed milk diluted with PBS-T. The membrane was incubated overnight with selected human sera diluted with blocking buffer ( $5 \mathrm{x}$ dilution) at $4^{\circ} \mathrm{C}$. Then the membrane was incubated with biotinylated anti-human IgE (Sigma-Aldrich, Saint Louis, MO, USA) for 1 hour. Finally, the membrane was incubated with peroxidase-conjugated ExtrAvidin (Sigma-Aldrich, Saint Louis, MO, USA) for 1 hour. The membrane was washed three times with PBS-T between steps. Results were detected by incubation with Alkaline Phosphatase Color Development Solution (BioRad).

\section{Statistical analysis}

Data were analyzed using statistical packages SAS9.2, STATA, GraphPad Prism 6, SPSSv16 and MS excel. (Microsoft Corp., Redmond, WA, USA). Statistical significance was defined as $p$-value is $<0.05$.

\section{Results}

One hundred twenty five match-paired study subjects were recruited for the study with a mean age of $8.34 \pm 3.90$ years ranging from 6 months to 18 years old. Most of the qualified subjects were male and aged 7-10 years old. Most of the allergic asthma children were not exposed to smoking environment and received Bacillus Calmette-Guérin (BCG) immunization. Their family history of asthma reported that most of them had both their parents and sibling suffering from asthma. Parental history of asthma was observed more frequently than maternal history (Table 1).

Total IgE levels in allergic asthma cases are significantly elevated $(t=16.08, d f=124, p<0.0001)$ compared to non-atopic controls (For figure refer to Philippine Science Letter, 6, 241-248; 2013). The mean total IgE concentration of the allergic asthma cases (247.52) is notably higher and is almost eight times than the non-atopic controls (32.89). Total IgE concentration of allergic asthma cases ranges from 100-1700 $\mathrm{IU} / \mathrm{mL}, 20 \%(30 / 125)$ had $<200 \mathrm{IU} / \mathrm{mL}$. In addition, a significant difference between the two means of the allergic asthma cases and non-atopic control group of the $\log \mathrm{HDM}$-specific $\operatorname{IgE}(\mathrm{IU} / \mathrm{mL})$ was observed in each dust mite species tested (Blomia tropicalis, $\mathrm{t}=20.20$, $\mathrm{df}=124, \mathrm{p}<0.0001 ;$ Dermatophagoides pteronyssinus, $\mathrm{t}=16.37, \mathrm{df}=124$, $\mathrm{p}<0.0005$; Dermatophagoides farinae, $\mathrm{t}=18.48, \mathrm{df}=124, \mathrm{p}<0.0001$ ). 
Across three HDM species tested, an evident difference in the means of the allergic asthma cases $(B t=91.83, D f=80.81, D p=80.09)$ and nonatopic controls ( $B t=2.74, D f=2.98, D p=4.19)$ were observed. HDMspecific IgE means of allergic asthma cases ranges from less than 50 to $1500 \mathrm{IU} / \mathrm{mL}$. Thirty five percent (44/125) of the Bt- specific IgE concentration was greater than $100 \mathrm{IU} / \mathrm{mL}$. Dp-specific IgE concentration registered the highest reading at $1587 \mathrm{IU} / \mathrm{mL}$ and $26 \%$ $(33 / 125)$ registered to have $<100 \mathrm{IU} / \mathrm{mL}$. Thirty three percent $(41 / 125)$ of the allergic asthma cases had results of 50-100 IU/mL Df-specific IgE concentration and 30\% (37/125) had results higher than 100 $\mathrm{IU} / \mathrm{mL}$.

\begin{tabular}{|c|c|c|c|}
\hline & & $\begin{array}{l}\text { Allergic Asthma } \\
\text { Cases }(n=125)\end{array}$ & $\begin{array}{l}\text { Non-atopic } \\
\text { Controls } \\
(n=125)\end{array}$ \\
\hline Age & Mean \pm SD & $8.34 \pm 3.90$ & $8.6 \pm 4.08$ \\
\hline & Female & $43(34.4 \%)$ & $43(34.4 \%)$ \\
\hline Sex & Male & $82(65.6 \%)$ & $82(65.6 \%)$ \\
\hline Environment & Yes & $31(24.8 \%)$ & 60 \\
\hline $\begin{array}{l}\text { Smoke } \\
\text { Exposure }\end{array}$ & No & $93(74.4 \%)$ & 65 \\
\hline \multirow[b]{6}{*}{$\begin{array}{l}\text { Family History } \\
\text { of asthma }\end{array}$} & Maternal Only & $7(5.6 \%)$ & \multirow{6}{*}{$N A^{1}$} \\
\hline & Paternal Only & $14(11.2 \%)$ & \\
\hline & Both Parents & $11(8.8 \%)$ & \\
\hline & Sibling Only & $17(13.6 \%)$ & \\
\hline & $\begin{array}{l}\text { Parental and } \\
\text { Sibling }\end{array}$ & $60(48 \%)$ & \\
\hline & $\begin{array}{l}\text { None of the family } \\
\text { members }\end{array}$ & $16(12.8 \%)$ & \\
\hline \multirow{2}{*}{$\begin{array}{l}\text { BCG } \\
\text { immunization }\end{array}$} & Yes & $111(88.8 \%)$ & \multirow{2}{*}{$N D^{2}$} \\
\hline & No & $5(4 \%)$ & \\
\hline
\end{tabular}

Table 1: Demographic profiles of the 125 allergic asthma cases and 125 non-atopic controls

Allergens form HDM species Bt, Df, and Dp triggered elevated IgE production in allergic asthma cases. Using a cut-off value of 8.30 , $10.35,11.50$ for Bt, Df, and Dp respectively, which corresponds to the mean +1 SD of NA, more than $60 \%$ of the allergic asthma cases reacted positively (For figure refer to Philippine Science Letter, 6, 241-248; 2013).

Detailed analysis of the profile of IgE reactivity of the 125 allergic asthmatic cases showed multiple sensitivity to different allergens of the three HDMs. Reactions to $\mathrm{Bt}, \mathrm{Df}, \mathrm{Dp}$ alone showed that most of the cases were sensitized to Bt (23/125) followed by Dp (16/125) and Df $(12 / 125)$. On the other hand, highest double positive reactions were observed between Bt and Df (20/125) followed by reactions to Dp and Df (14/125) and Bt and Dp (8/125).

Furthermore, 26\% (32/125) of the patients tested showed triple positive IgE- binding positive reactions to $\mathrm{Bt}$, Df, and $\mathrm{Dp}$ allergens (Figure 1).

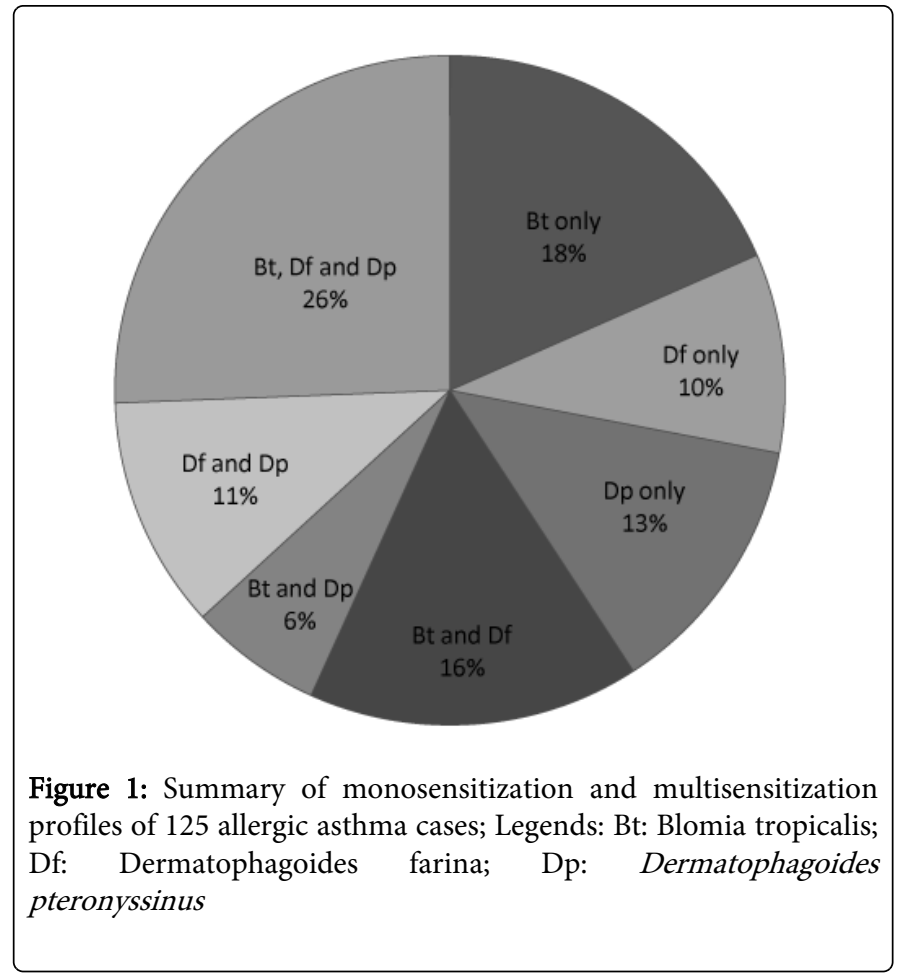

\section{IgE reactivity of three $\mathrm{HDM}$}

Different protein bands were obtained from the three HDM extracts using SDS-PAGE. Protein bands ranging from approximately 20-150 $\mathrm{kDa}$ from the three HDM extracts were observed under $15 \%$ Trisglycine gel. Western blot analysis was used to examine the $\operatorname{IgE}$ reactivity of the patients' sera that binds to the protein bands observed in SDS-PAGE. Five sera samples that resulted positive reactions to the three HDM species as determined by ELISA were selected for IgEbinding activity. Multiple serum IgE reacted to different proteins in the HDM aqueous extract with molecular weight ranging from 20-100 $\mathrm{kDa}$. Majority of patients' IgE reacted to an approximately $70 \mathrm{kDa}$ protein in the Bt extract. On the other hand patients' IgE reacted to an approximately $60 \mathrm{kDa}, 80 \mathrm{kDa}$ protein in the $\mathrm{Dp}$ and Df extract respectively. It was also observed that there are minor IgE-reactive protein bands of $<25 \mathrm{kDa}$, and approximately $30 \mathrm{kDa}, 40 \mathrm{kDa}, 50 \mathrm{kDa}$, $60 \mathrm{kDa}$, and $70 \mathrm{kDa}$ among the three HDM species (Figure 2). These IgE reactive proteins contribute to the allergic sensitization among local asthmatic children tested.

\section{Correlation between total IgE and HDM-specific IgE}

Correlation between total serum IgE and HDM-specific IgE was also tested in the study (Table 2). Significant correlation between total IgE and the three HDM (Bt $\mathrm{p}$-value $=0.038 ; D f \mathrm{p}$-value $=0.045 ; D p \mathrm{p}$ value $=0.003)$ was observed among the allergic asthmatic patients. Furthermore, the study also analyzed the correlation among HDM specific IgE (Table 2). Patients with allergic asthma showed a significant correlation between $B t$ and $D f$ (p-value $<0.0001)$ as well as between $D f$ and $D p$ (p-value $<0.0001)$. Using multiple regression model $D f$ and $D p$ IgE concentration was significantly contributing to the increase of total IgE concentration. This means that for every one unit increase in $D f$ (parameter estimate $=1.37$, test stat $=3.11$, $\mathrm{p}=0.0023$ ), on the average, the total $\operatorname{IgE}$ concentration is expected to 
increase by 1.37 controlling for $B t$ and $D p$. The coefficient of $B t$ is also positive but not significant (parameter estimate $=0.33$, test stat $=1.33$, $\mathrm{p}$ value $=0.1857$ ). This means that an increase in $B t$ is expected to increase the average total IgE concentration.

\begin{tabular}{|c|c|c|c|c|}
\hline & & $\begin{array}{l}\text { Correlation } \\
\text { value }\end{array}$ & P-value & Remarks \\
\hline \multirow{3}{*}{$\begin{array}{l}\text { Total } \\
\text { IgE vs. } \\
\text { HDM- } \\
\text { specific } \\
\text { IgE }\end{array}$} & $\begin{array}{l}\text { Total lgE vs Bt- specific } \\
\text { lgE }\end{array}$ & 0.185 & 0.038 & $\mathrm{~S}^{1}$ \\
\hline & $\begin{array}{l}\text { Total IgE vs Df- specific } \\
\text { lgE }\end{array}$ & 0.18 & 0.045 & $\mathrm{~s}$ \\
\hline & $\begin{array}{l}\text { Total lgE vs Dp-specific } \\
\text { IgE }\end{array}$ & 0.261 & 0.003 & $\mathrm{~S}$ \\
\hline \multirow{3}{*}{$\begin{array}{l}\text { HDM- } \\
\text { specific } \\
\text { IgE }\end{array}$} & $\begin{array}{l}\text { Bt- specific lgE vs Df- } \\
\text { specific } \lg E\end{array}$ & 0.404 & $<0.0001$ & $\mathrm{~s}$ \\
\hline & $\begin{array}{l}\text { Bt- specific IgE vs Dp- } \\
\text { specific } \lg E\end{array}$ & 0.146 & 0.105 & $\mathrm{NS}^{2}$ \\
\hline & $\begin{array}{l}\text { Df- specific lgE vs Dp- } \\
\text { specific lgE }\end{array}$ & 0.455 & $<0.0001$ & $s$ \\
\hline
\end{tabular}

Table 2: Correlation between total IgE and HDM-specific IgE in 125 allergic asthma cases

HDM: House Dust Mite; Bt: Blomia tropicalis; Df: Dermatophagoides farinae, Dp: Dermatophagoides pteronyssinus. ${ }^{1} \mathrm{~S}$ : significant; ${ }^{2} \mathrm{NS}$ : not significant

\section{Discussion}

The prevalence of allergic diseases such as allergic asthma, allergic rhinitis, and atopic dermatitis is increasing across all populations especially among children [12]. Hence, it is imperative to evaluate potential risk factors to these diseases. An important factor to be considered in the development of asthma is the influence of exposure to house dust mite allergens [13]. Different studies confirmed that sensitization to indoor allergens such as house dust mites are major risk factors in allergy, especially in asthma [14]. Three dust mite species were used in this study to determine the sensitization profile of the Filipino asthmatic children. Common house dust mites implicated in allergic diseases, such as Blomia tropicalis (Bt), Dermatophagoides farinae (Df) and Dermatophagoides pteronyssinus (Dp), are considered ubiquitous in every household, preferably in areas with high relative humidity ( $>45 \%$ ) and warm temperatures, between 65 to $85^{\circ} \mathrm{F}$ [15]. B. tropicalis, which is morphologically different from the other dust mites, is geographically distributed in tropical and subtropical regions, although can also be found in temperate countries. It has been traditionally referred to as "storage mites" because they have been found mainly in stored grain. Dermatophagoides species, commonly known as the "house dust mites," are predominantly found in temperate and tropical regions. $D$. farinae and $D$. pterynissinus are the most common species which are widely distributed worldwide. Various studies have reported double sensitization involving $B$. tropicalis and $D$. pterynissinus [10,16-19]. Results of this study showed that $B$. tropicalis is responsible for the sensitization of $66 \%(83 / 125)$ allergic asthmatic Filipino population. This is consistent with the reports of very high sensitivity to $B$. tropicalis among asthmatic population in other countries in Asia such as in Taiwan [18], Singapore and Malaysia [16]. High prevalence and high variability of $B$. tropicalis was also observed among atopic subjects in (40\%) India and in (82\%) Indonesia [18]. Surprisingly, $D$. pteronyssinus obtained the lowest reactivity at $56 \%$ and $D$. farinae at $62 \%$.

Further analysis of the sensitization profile also showed that, patients with allergic asthma (16\%) were sensitized to both $B$. tropicalis and $D$. farinae. The result of double sensitization to $B$. tropicalis and $D$. farinae is consistent with the report of Ramos et al, which showed $5 \%(11 / 210)$ double positive reactions to B. tropicalis and $D$. farinae, as compared to $3 \%(7 / 210)$ and $2 \%(4 / 210)$ sensitization to $B$. tropicalis and $D$. pteronyssinus, and $D$. pteronyssinus and $D$. farinae, respectively. This is not surprising, considering that the climatic conditions in a tropical country like the Philippines and the fact that $D$. farinae is more able to survive in drier climatic conditions than $D$. pteronyssinus, which prefers consistently damp conditions [11]. A previous work investigating the acrofauna in houses of allergic patients in Metro Manila (Philippines) revealed that Dermatophagoides and Blomia species are the most abundant HDM species [20]. Our results showed that $26 \%$ of the asthmatic patients were sensitized to the three dust mites tested, indicative of moderate prevalence of house dust mite sensitization among Filipino allergic asthmatic patients. Different house dust mite species can possibly coinhabit homes and dust mites allergens may cause parallel allergic sensitization among susceptible individuals [11].

Our results showed significant correlation between $D$. farinae and $D$. pteronyssinus, and between $D$. farinae and B. tropicalis. These results support the double positive reactions between Dp and Df, as well as in Df and Bt which have an average reaction of 13\% (Table 2). Significant correlation may indicate that as the level of Df-specific IgE increases, the level of Dp-specific IgE also increases. The observed multiple specific IgE reactivities can be attributed to the presence of cross-reactive allergens from different HDM species.

Sensitization to different house dust mite allergens showed that patients who have asthma and atopic dermatitis have highest sensitization rate to $D$. farinae and $D$. pteronyssinus in 2 out of 3 HDM species tested and to all three HDM species tested (data not shown). This is consistent with the result of a study by Shek et al, wherein patients with eczema were generally more sensitized to the panel of dust mite allergens, indicating susceptibility to HDM. In another study, it was observed that eczema in the first 3 months of life was a risk factor for sensitization to aeroallergens by 5 year of age and subsequent manifestation of respiratory allergies [21]. Moreover, another study showed that children with eczema were significantly more sensitized to a panel of allergens tested than those without skin diseases. The degree of sensitization was also observed to be directly associated with the severity of eczema, especially for HDM allergens [22].

In this study, asthmatic patients showed high sensitivity to Bt. This is similar with the results of a study by Shek et al. which reported that asthmatics are more sensitized to $B$. tropicalis, more specifically to Blot 5 allergen. In Singapore, there is also a higher reactivity observed in $B$. tropicalis among HDM- sensitized patients [10]. Likewise, Manolio [23] reported that $B$. tropicalis sensitization was more prevalent than $D$. pteronyssinus sensitization and that $D$. pteronyssinus sensitization without $B$. tropicalis sensitization was uncommon. In addition, the study found that dual sensitization to $D$. pteronyssinus and $B$. tropicalis resulted in higher risk of asthma.

In previous reports of sensitization involving HDM species, it is notable that patients with skin allergies are found to be more 
sensitized to D. pteronyssinus while individuals with respiratory allergies are more sensitized to $\mathrm{B}$. tropicalis. Protease activities of allergens from D. pteronyssinus can influence allergenicity through several mechanisms such as causing disruption of the epithelium, allowing access of allergens to antigen-presenting cells, activation of complement and kallilkrein, and induction of inflammatory reactions, thus possibly aggravating skin allergies upon contact [24]. Molecular weight of Der p 1, which is a major allergen of D. pteronyssinus, is 25 $\mathrm{kDa}$ cysteine protease carried by large particles of median size $25 \mathrm{~mm}$ $[16,25]$. Because of their size, these particles do not stay airborne, and deposit rapidly upon skin contact. It may also be the absence of Der p1 protease inhibitor in sweat, distinct to patients with eczema, which increases their susceptibility to D. pteronyssinus sensitization [16]. B. tropicalis allergens are noted for their proteolytic activity, lipid binding activity, chitin- binding activity, muscle proteins, and allergens without autoadjuvant or adjuvant activity. Chitinase is known to play an important role in the pathogenesis of asthma [24]. In addition, that the major allergen of $\mathrm{B}$. tropicalis which is Blo $\mathrm{t} 5$ has a molecular weight only of $14 \mathrm{kDa}$ thus can remain airborne longer resulting in airway sensitization [16]. The IgE binding reactivity among the three HDM species tested confirmed that proteins from HDM contribute to the allergic sensitization of asthmatic patients and that exposure to these allergens may contribute to the pathogenesis of asthma.

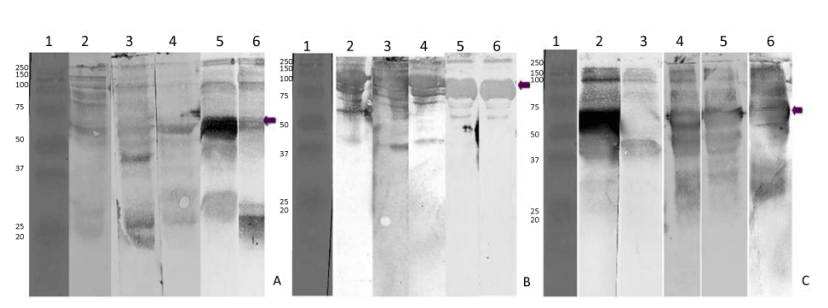

Figure 2: Western blot analysis showing the IgE reactivity of major protein bands from house dust mite aqueous extracts using five allergic patients' sera. A: Blomia tropicalis extract; B: Dermatophagoides farina extract; and C: Dermatophagoides pteronyssinus extract. Lane 1: protein marker (BioRad Pre-stained Standards Broad Range) while lanes 2-6: different sera from selected allergic patients. Arrow A: majority of patient's IgE reacted to $\sim 70 \mathrm{kDa}$ protein; Arrow B: majority of patient's IgE reacted to $\sim 80 \mathrm{kDa}$ protein; Arrow C: majority of patient's IgE reacted to $\sim 60 \mathrm{kDa}$ protein.

The results of this study are consistent with the results of previous studies that showed significantly elevated total and HDM-specific IgE levels among allergic patients as compared with the controls (Figures 1 and 2) [26]. It is well established that specific serum IgE antibody can be quantitatively high, relative to the total serum IgE. It could also be noted that higher serum IgE is related to the prevalence and severity of asthma and other allergic disease [27]. Any assay for specific IgE antibody is inevitably a conservative estimate because it is dependent on the affinity of the antibody for the allergen and that the assay for total IgE uses high affinity antibodies for the isotype-specific epitopes on the heavy chain and is unlikely to underestimate total IgE [28]. Based on our results, the highest correlation value was observed between total IgE and HDM- specific IgE level among allergic asthmatics. Thus it can be said that HDM sensitization could have possibly contributed to the elevation of total IgE level among allergic asthmatic patients. This finding validates the use of specific IgE measurement in the diagnostic and therapeutic strategies for housedust mite induced asthma.

Results of this study confirm that the total serum IgE and HDMspecific IgE are significantly correlated among allergic asthmatic Filipino paediatric population. Sensitization to B. tropicalis and $D$. farinae is more prevalent among allergic asthmatics and those with concurrent allergic rhinitis, while sensitization to $D$. farinae and D.pteronyssinus is significant only among asthmatics with atopic dermatitis but not in patients with asthma alone. Incorporation of the three house dust mite allergens in the panel of diagnostic and immunotherapeutic allergens for allergic paediatric population is highly recommended.

\section{Acknowledgements}

This work was supported by research grants from the Department of Science and technology- Accelerated Science and Technology Human Resource Development Program and the Commission on Higher Education-Zonal Research Center. The researchers would like to acknowledge the Research Center for the Natural Sciences (RCNS) for housing the experiment, and the Philippines Children Medial Center Ethics Review Board for reviewing our protocol. We would also like to thank Mr. Kevin Carl Santos of University of the Philippines for his assistance in the statistical analysis of data.

\section{References}

1. Hellman L (2007) Regulation of IgE homeostasis, and the identification of potential targets for therapeutic intervention. Biomed Pharmacother 61: 34-49.

2. Baxi SN, Phipatanakul W (2010) The role of allergen exposure and avoidance in asthma. Adolesc Med State Art Rev 21: 57-71, viii-ix.

3. Wickens K, Martin I, Pearce N, Fitzharris P, Kent R, et al. (1997) House dust mite allergen levels in public places in New Zealand. J Allergy Clin Immunol 99: 587-593.

4. Noguchi E, Ohtsuki Y, Tokunaga K, Yamaoka-Sageshima M, Ichikawa K, et al. (2006) ADAM33 polymorphisms are associated with asthma susceptibility in a Japanese population. Clin Exp Allergy 36: 602-608.

5. Platts-Mills T, Leung DY, Schatz M (2007) The role of allergens in asthma. Am Fam Physician 76: 675-680.

6. Lodge CJ, Lowe AJ, Gurrin LC, Hill DJ, Hosking CS, et al. (2011) House dust mite sensitization in toddlers predicts current wheeze at age 12 years. J Allergy Clin Immunol 128: 782-788.

7. Cheong N, Soon SC, Ramos JD, Kuo IC, Kolatkar PR, et al. (2003) Lack of human IgE cross-reactivity between mite allergens Blo 1 and Der $\mathrm{p} 1$. Allergy 58: 912-920.

8. Valdivieso R, Iraola V, Estupiñán $M$, Fernández-Caldas E (2006) Sensitization and exposure to house dust and storage mites in highaltitude areas of ecuador. Ann Allergy Asthma Immunol 97: 532-538.

9. Sidenius KE, Hallas TE, Poulsen LK, Mosbech H (2001) Allergen crossreactivity between house-dust mites and other invertebrates. Allergy 56: 723-733.

10. Kuo IC, Yi FC, Cheong N, Shek LP, Chew FT, et al. (1999) Sensitization to Blomia tropicalis and dermatophagoides pteronyssinus-a comparative study between Singapore and Taiwan. Asian Pac J Allergy Immunol 17: 179-188.

11. Ramos JD, Castillo MP, del Rosario M, Gapay MA, Go TP, et al. (2007) Allergenicity and Cross-Reactivity of 3 House Dust Mites Among Filipino Allergic Patients. Philipp J Sci 136: 139-146.

12. Shin JW, Sue JH, Song TW, Kim KW, Kim ES, et al. (2005) Atopy and house dust mite sensitization as risk factors for asthma in children. Yonsei Med J 46: 629-634. 
Citation: Yap JMG, Ching MW, Cabanilla CQ, Ramos JDA (2014) Multiple House Dust Mite Allergen- Sensitization Profiles in Children with Allergic Asthma. J Allergy Ther 5: 179. doi:10.4172/2155-6121.1000179

Page 6 of 6

13. Baqueiro T, Russo M, Silva VM, Meirelles T, Oliveira PR, et al. (2010) Respiratory allergy to Blomia tropicalis: Immune response in four syngeneic mouse strains and assessment of a low allergen-dose, shortterm experimental model. Respir Res 11: 51-62.

14. Platts-Mills TA, Rakes G, Heymann PW (2000) The relevance of allergen exposure to the development of asthma in childhood. J Allergy Clin Immunol 105: S503-508.

15. Milián E, Díaz AM (2004) Allergy to house dust mites and asthma. P R Health Sci J 23: 47-57.

16. Shek LP, Chong AR, Soh SE, Cheong N, Teo AS, et al. (2010) Specific profiles of house dust mite sensitization in children with asthma and in children with eczema. Pediatr Allergy Immunol 21: e718-722.

17. Thomas WR, Hales BJ, Smith WA (2010) House dust mite allergens in asthma and allergy. Trends Mol Med 16: 321-328.

18. Tsai JJ, Yi FC, Chua KY, Liu YH, Lee BW, et al. (2003) Identification of the major allergenic components in Blomia tropicalis and the relevance of the specific IgE in asthmatic patients. Ann Allergy Asthma Immunol 91: 485-489.

19. Chew FT, Yi FC, Chua KY, Fernandez-Caldas E, Arruda LK, et al. (1999) Allergenic differences between the domestic mites Blomia tropicalis and Dermatophagoides pteronyssinus. Clin Exp Allergy 29: 982-988.

20. Ramos JD, Concepcion ZS, Pineda BA, Romano EL, Saep JB, et al. (2006) Acarofauna and allergenicity of house dust mites in houses of selected Filipino allergic patients in Metro Manila. Acta Manila Ser A 54: 7-14.

21. Bergmann RL, Edenharter G, Bergmann KE, Forster J, Bauer CP, et al. (1998) Atopic dermatitis in early infancy predicts allergic airway disease at 5 years. Clin Exp Allergy 28: 965-970.
22. Schäfer T, Heinrich J, Wjst M, Adam H, Ring J, et al. (1999) Association between severity of atopic eczema and degree of sensitization to aeroallergens in schoolchildren. J Allergy Clin Immunol 104: 1280-1284.

23. Manolio TA, Barnes KC, Naidu RP, Levett PN, Beaty TH, et al. (2003) Correlates of sensitization to Blomia tropicalis and Dermatophagoides pteronyssinus in asthma in Barbados. Int Arch Allergy Immunol 131: 119-126.

24. Jeong KY, Park JW, Hong CS (2012) House dust mite allergy in Korea: the most important inhalant allergen in current and future. Allergy Asthma Immunol Res 4: 313-325.

25. De Lucca S, Sporik R, O'Meara TJ, Tovey ER (1999) Mite allergen (Der p 1 ) is not only carried on mite feces. J Allergy Clin Immunol 103: 174-175.

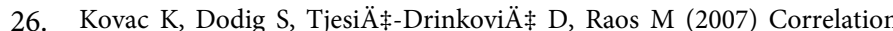
between asthma severity and serum IgE in asthmatic children sensitized to Dermatophagoides pteronyssinus. Arch Med Res 38: 99-105.

27. Naqvi M, Choudhry S, Tsai HJ, Thyne S, Navarro D, et al. (2007) Association between IgE levels and asthma severity among African American, Mexican, and Puerto Rican patients with asthma. J Allergy Clin Immunol 120: 137-143.

28. Erwin EA, Rönmark E, Wickens K, Perzanowski MS, Barry D, et al. (2007) Contribution of dust mite and cat specific IgE to total IgE: relevance to asthma prevalence. J Allergy Clin Immunol 119: 359-365. 